\section{MRS expands materials publications landscape}

\author{
www.mrs.org/mrs-publishing
}

T he publishing image and landscape with regard to MRS involvement is changing. "It is not just the old blue book set anymore," said Steven C. Moss, a member of the New Publications Products Subcommittee at the Materials Research Society. Since partnering with Cambridge University Press, MRS has been focusing on publishing high-quality scientific books and book series, available in a variety of formats, in print as well as electronically. Most importantly for materials researchers, this partnership has opened a new avenue to authoring a book or spearheading a new journal.

Betsy Fleischer, MRS Principal Development Editor, is the staff liaison to the Subcommittee. As a materials scientist herself with connections to the materials community, she oversees the review of publication proposals in conjunction with the New Publications Products Subcommittee and Cambridge University Press. Information on how to propose a new book or journal is prominently displayed on the MRS website.

Cambridge University Press as a publisher of high-quality technical books - that also have a professional and appealing layout and design - brings international recognition to the table, as well as streamlined editorial approaches and methods. MRS, on the other side, has great experience in promoting the exchange of cutting-edge research and attracting world-class researchers to join them in this endeavor. The partnership combines everything that is needed to publish influential technical books of value to materials researchers.

A Selection of

Current and Upooming

Books and Journals

www.mis: org/publications

Fundamentals of Materials for Energy and Environmental Sustainability Editors: David S. Ginley and David Cahen

Introduction to Computational Materials Science: Fundamentals to Applications Author: Richard LeSar

Colloidal Quantum Dot Optoelectronics and Photovoltaics

Editors: Gerasimos Konstantatos and Edward H. Sargent

MRS Communications www.mrs.org/mrc

MRS Energy \& Sustainability-

A Review Journal

www.mrs.org/energy-sustainability-journal
Texts already on the market as a part of this partnership include Richard LeSar's Introduction to Computational Materials Science: Fundamentals to Applications and editors Gerasimos Konstantatos and Edward H. Sargent's book Colloidal Quantum Dot Optoelectronics and Photovoltaics. The vast majority of journal articles on either topic have been published within the last 10 years, and their number is still increasing in year-by-year comparisons. Books available on computational methods tend to focus on a specific method or on a narrow range of phenomena or applications, which can be investigated by theoretical means. The timeliness and value to the materials community of LeSar's textbook, however, is that it is one of only a handful of textbooks that addresses the breadth of materials science.

The partnership between MRS and Cambridge University Press will not be limited to just publishing books. MRS Energy \& Sustainability-A Review Journal is a new journal in their pipeline. The first issue will come out later this year. One goal of the new journal is to disseminate reviews on current energy- and sustainability-related topics in materials research and development beyond the traditional readership target of scientists and academics. This journal aims to reach policymakers and industry professionals as well. David S. Ginley (National Renewable Energy Laboratory, USA), David Cahen (Weizmann Institute of Science, Israel), and Sally M. Benson (Stanford University, USA), as Editors-in-Chief of the new journal, will be supported by a similarly illustrious advisory board in this quest. The new journal will join the current portfolio of MRS Bulletin, Journal of Materials Research, MRS Communications, and MRS Online Proceedings Library.

Richard A. Vaia, chair of the Subcommittee, said that the New Publications Products Subcommittee is welcoming inquiries. The Subcommittee looks toward the materials community to identify emerging research areas meriting publication.

Birgit Schwenzer 
2-DTech Ltd.

Booth 427 www.2-dtech.com

Balazs NanoAnalysis, a Division

of Air Liquide Electronics U.S. LP

Graphene; 2-D Materials; Graphene 0xide; Consultancy

A \& N Corporation

Booth 316

www.ancorp.com

Vacuum Components; Vacuum Chambers; Vacuum Valves

ACS Publications

Booth 606

pubs.acs.org

ACS Nano: Nano Letters; Journal of Physical Chemistry C.

ACS Applied Materials \& Interfaces; ACS Photonics

Active Spectrum, Inc.

Booth 519

www.activespectrum.com

Benchtop Micro-ESR; Carbon-X Nano Carbon;

FISE Ion Sensors

AdValue Technology, LLC

WWw.advaluetech.com

Alumina and Quartz Crucibles, Tubes and Plates;

Quartz Cuvettes; Sapphire Crucibles

Advanced Research Systems, Inc.

Www.balazs.com

Analytical Testing; Materials Characterization; AMC-SMC

Barnett Technical Services LLC

www.barnett-technical.com

Scanning Probe Microscopes; Cathodoluminescence

Systems; Micro-manipulators

Bio-Logic USA, LLC

www.bio-logic.us

Potentiostat; Battery Cycler; Scanning Probe Microscopy

Blue Wave Semiconductors, Inc.

www.bluewavesemi.com

Substrate Wafer Heaters; Thin Film Deposition Systems;

Thin Films and Coating Materials; R\&D Services

Brookhaven Instruments Corporation Booth 531

www.brookhaveninstruments com

Particle Size Analyzers: Zeta Potential Analyzers: Molecula

Weight Analyzers

\section{Bruker}

www.arscryo.com

4K Cryocoolers; Custom Lab Systems; Nanoscience

Materials Characterization

AIP Publishing

journals.aip.org

Physics Journals; Conference Proceedings; Digital Archive

AIST-NT, Inc.

www.aist-nt.com

Atomic Force/Scanning Probe Microscopes; Combined AFM and Raman Spectroscopy Systems

\section{AIXTRON SE}

Www.aixtron.com

MOCVD/CVD/PECVD Equipment; OVPD and PVPD

Equipment; ALD Equipment

AJA International, Inc.

Www.ajaint.com

Sputtering Systems; Sputter Sources; Sputter Targets

Aldrich Materials Science

www.sigmaaldrich.com/matsci

Organic and Printed Electronics Materials: Nanomaterials;

Materials for Energy and Electronics

American Physical Society

ournals.aps.org

Publications; Physics Journals; Online Journals

Angstrom Scientific Inc.

www.angstrom.us

TEM Heating Holders

Angstrom Thin Film Technologies LLC

www.angstrom-dep.com

Atomic Layer Deposition System; Plasma-ALD System;

Powder ALD System

Annealsys

www.annealsys.com

RTP; MOCVD; ALD; RTCVD; LPCVD

Arradiance, Inc.

www.arradiance.com

GEMStarTM ALD Systems; ALD Coating Services;

ALD-Activated Microchannel Plates

Asahi Spectra Co., Ltd.

www.asahi-spectra.com

Xenon Light Source: Monochromatic Illuminator; Optical Filters

Asylum Research, an 0xford

Instruments Company

www.AsylumResearch.com

Atomic Force/Scanning Probe Microscopes;

AFM/SPM Probes
Booth 60

Booth 50

Booth 624

Booth 324

Booth 230

Booth 527

X-Ray Diffraction; Near Infrared Spectroscopy;

Raman Spectroscopy

Cambridge University Press

www.cambridge.org/us/academic

Booth 611

Books; Journals

Carl Zeiss X-Ray Microscopy, Inc.

Booth 100

Xradia Ultra; Xradia Versa; SteREO Discovery V20

CellScale Biomaterials Testing

Booth 328

www cellscale com

Biomaterials Testing Equipment; Custom Testing Equipment; Biological Stimulation Equipment

Chemat Technology, Inc.

Booth 101

www.chemat.com

Spin Coating; Dip Coating; Chemical Precursors

CRAIC Technologies, Inc.

Www.microspectra.com

UV-visible-NIR microscopes; Microspectrophotometers;

Raman Microspectrometers

\section{CRC Press}

www.crcpress.com

Books; Electronic Content

CrystalMaker Software Ltd.

www.crystalmaker.com

CrystalMaker; CrystalDiffract; SingleCrystal

www.cvdequipment.com

CVD Equipment; Gas/Chemical Delivery Systems;

Gas Abatement Systems

www.duniway.com

Vacuum Pumps; Vacuum Gauges; Vacuum Supplies

Ecopia Corp.

www.ecopia21.co.kr

EDP Sciences

publications.edpsciences.org

Physical Journals, Scientific Articles

\section{Effucell}

www.effucell.com

UHV Heater; Multiple Effusion Cell

Electron Microscopy Sciences/Diatome

Grinders/Polishers; Diamond Wheels/Saws;

Grinders/Polishers; D
Microscopy Supplies

EnvisionTEC, Inc.

WwW.envisiontec.com

3D-Bioplotter

Booth 620 www.zeiss.com $\backslash X R M$

CVD Equipment Corporation

Duniway Stockroom Corporation

Hall Effect Measurement Systems; RTP Systems

www.emsdiasum.com
Booth 318

Booth 401

Fischione Instruments $\quad$ Booth 404

www fischione com

TEM Accessories; Sample Preparation; Specimen Holders

FUJIFILM Dimatix, Inc.

Booth 431

www.dimatix.com

Dimatix Materials Printer; Dimatix Materials Cartridge;

Inkjet Printheads \& Systems

Gamry Instruments

www.gamry.com

Potentiostats: Impedance Spectroscopy; Quartz Crystal

Microbalance

Gatan, Inc.

www.gatan.com

Materials Characterization; Nanotechnology; Photovoltaics

Gencoa Ltd.

Booth 117

www.gencoa.com

Magnetrons; Reactive Gas Controllers; Ion Sources

HeatWave Labs Inc.

www.cathode.com

Booth 505

Substrate Heaters; Cathodes; Electron Gun Assemblies

HORIBA Scientific

www horiba.com/scientific

Booth 507

Materials Characterization; Microscopy; Nanotechnology;

Optical Components; Spectroscopy; Surface Analysis;

Ellipsometry

Hummingbird Scientific

www.humming birdscientific.com

Booth 212

In Situ TEM Holders; TEM Holders; Environmental Holders

Huntington Mechanical Laboratories, Inc. Booth 300 www.huntvac.com

Vacuum Manipulation \& Positioning; Vacuum Chambers;

Vacuum Valves \& Components

Hysitron, Inc.

Booth 410

www hysitron.com

TI 950 Tribolndenter; PI 95 TEM Picolndenter; TI 750 Ubi

ICE Publishing

Booth 618

www.icevirtuallibrary.com

ICE Science Journals

Integrated Dynamics Engineering

Booth 330

www.ideworld.com

Vibration Isolation; EMI Cancellation; Acoustic Control

International Centre for Diffraction

Data (ICDD)

www icdd com

X-Ray Powder Diffraction; Database; Software

IOP Publishing

ioppublishing.org

Journals, magazines and ebooks covering materials

research, applied physics, nanoscience and novel

applications

Janis Research Company, LLC

Booth 501

WWW.janis.com

Micromanipulated Probe Stations; Cryostats; Cryocoolers

Japan Society of Applied Physics

www.jsap.or.jp/english/index.html

Journals

JASCO

wiwijasco

Raman; Portable Raman; FTIR Microscopes; Near Field

Microscopy; Ellipsometry

JEOL USA, Inc.

Www.jeolusa.com

TEM; SEM; Auger/MicroProbe

JSOL Corporation

www.j-octa.com

J-OCTA Integrated Simulation System for Materials

Research \& Development

KP Technology USA Inc.

www.kelvinprobe.com

Air Photoemission, Scanning and UHV Kelvin Probe

Systems; Corrosion 


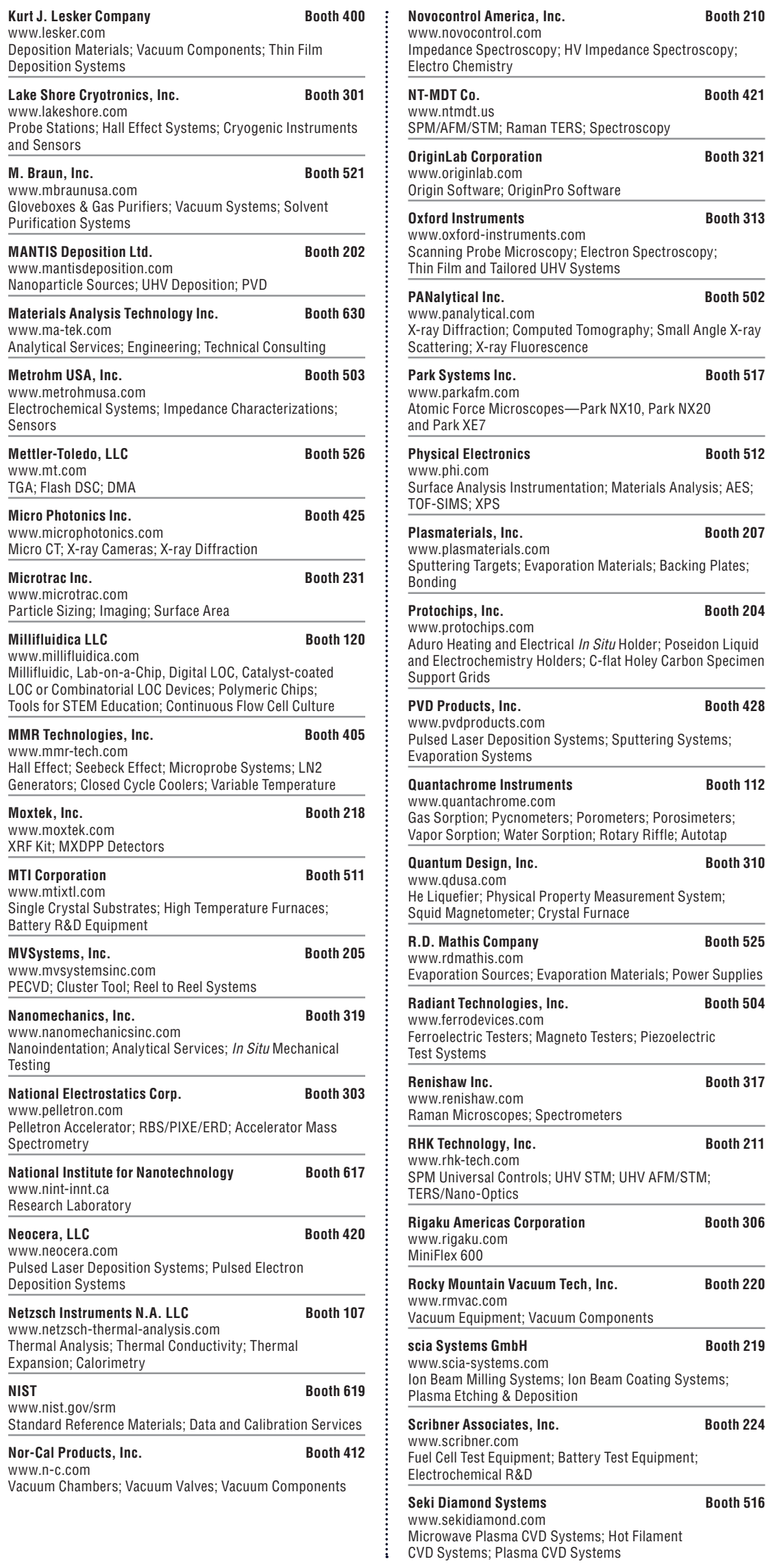

Semicore Equipment Inc.

www.semicore.com

Sputtering Systems; Evaporation Systems; Custom PVD Systems \& Components

Simpleware Ltd.

www.simpleware.com

Imaging Processing Software; Mesh Generation Software

Solartron Analytical (AMETEK) Booth 524

www.solartronanalytical.com

Materials Test Systems

SonoPlot, Inc.

www.sonoplot.com

Microplotter; Printed Electronics; Materials Printer

SPECS Surface Nano Analysis, Inc.

www.specs.com

JT Scanning Tunneling Microscope; NAP PHOIBOS Energy Analyzer; Curlew SPM

SPI Supplies/Structure Probe, Inc.

Booth 500

www.2spi.com

Plasma Etching Systems; Graphene Products; Ion Milling

Systems

Springer

Booth 601

www.springer.com

Books; Journals; E-Books

STAIB Instruments, Inc.

www.staibinstruments.com

RHEED; Auger; Surface Analysis

Strem Chemicals, Inc.

www.strem com

Nanomaterials; CVD/ALD Precursors; Bubblers

SVT Associates, Inc.

WWW.svta.com

Molecular Beam Epitaxy; Pulsed Laser Deposition;

Atomic Layer Deposition

Ted Pella, Inc.

www.tedpella.com

Microscopy Supplies; Sample Prep; Vacuum Coating

Tescan USA Inc.

www.tescan-usa.com

Scanning Electron Microscopes; Focused Ion Beam

Workstations; Mineral Analyzers

Thermo Scientific

www.thermoscientific.com/materialscience

Raman Microscope; XPS Spectrometer; X-Ray Microanalysis System

THINKY USA, Inc.

Booth 631

www.thinky-usa.com

Planetary Centrifugal Mixers; Nano-Pulverizers

United Mineral \& Chemical Corporation

Booth 111

www.umccorp.com

MBE Source Materials; MBE Equipment; Dopants

Vacuum Engineering \& Materials (VEM) Booth 113 www.vem-co.com

High Purity PVD Sputtering Targets; Elemental \& Composite High Purity PVD Sputtering Targets; Elemental \& Composite
Evaporation Materials; Materials Reclaim \& Shield Cleaning Services

Vacuum Technology Inc.

www.vti-glovebox.com

Glove Box; Gas Purification System; Evaporator

Verder Scientific, Inc.

www.verder-scientific.com

Laboratory Milling \& Sieving

Vigor Gas Purification Technologies Inc.

www.vigor-glovebox.com

Glove Box; Gas Purification System; Solvent Purification System

WITec Instruments Corp.

www.witec-instruments.com

Confocal Raman Microscopy; Scanning Near-Field

Microscopy; Atomic Force Microscopy

Zahner-elektrik GmbH \& Co. KG

www.zahner.de

Photo-electrochemical Workstations; Electrochemical

Workstations; Power-Potentiostats; Measurement/AnalysisSoftware 
